\title{
Editorial
}

Jessica Howard-Anderson, Andrew Webster*, Abhinav Goyal, David S. Vadnais, Colleen S. Kraft, James P. Steinberg and Jesse T. Jacob

\section{COVID-19 or common coronavirus? A cautionary tale in advanced diagnostics}

https://doi.org/10.1515/dx-2020-0093

The ongoing coronavirus pandemic has emphasized the importance of diagnostic medicine to both health professionals and the broader public. With rapid development and introduction of testing for SARS-CoV-2, much attention has been given to evaluating the reliability of these tests. However, as advanced diagnostics become increasingly used in healthcare worldwide, both performance characteristics of the test and the interpretation of results must be considered before implementation. Prior to widespread availability of SARS-CoV-2 testing, many clinicians used rapid viral diagnostics, including influenza PCR and multiplex respiratory PCR panels to look for evidence of alternative diagnoses in patients presenting with influenza-like illnesses. Common human coronaviruses (HCoV-229E, HCoV-OC43, HCoV-NL63 and HCoV-HKU1) which typically cause mild upper respiratory tract infections are targets in several commercial multiplex PCR panels, however none in March 2020, detected SARS CoV-2 (the cause of COVID-19) [1].

By mid-March 2020, >170,000 people in 150 countries had confirmed COVID-19 and patients who presented with respiratory complaints were commonly evaluated as persons under investigation (PUI) for COVID-19 [2]. During this time, we cared for a 58 year old male with non-ischemic cardiomyopathy and chronic obstructive pulmonary disease who presented to the hospital with dyspnea and

\footnotetext{
*Corresponding author: Andrew Webster, MD, Clinical Instructor, Emory University School of Medicine, 550 Peachtree Street Northeast, Medical Office Tower, Fl 7, Atlanta, GA, 30308, USA,

E-mail: awebst2@emory.edu

Jessica Howard-Anderson, James P. Steinberg and Jesse T. Jacob: Division of Infectious Diseases, Department of Medicine, Emory University School of Medicine, Atlanta, GA, USA

Abhinav Goyal and David S. Vadnais: Division of Cardiology, Department of Medicine, Emory University School of Medicine, Atlanta, GA, USA

Colleen S. Kraft: Division of Infectious Diseases, Department of Medicine, Emory University School of Medicine, Atlanta, GA, USA; Department of Pathology and Laboratory Medicine, Emory University School of Medicine, Atlanta, GA, USA
}

productive cough. He had an elevated jugular venous pressure, lower extremity edema and required intubation for hypercapnic respiratory failure and decompensated heart failure. After admission, he became febrile and a chest radiograph revealed bibasilar atelectasis. Because of recent locally reported COVID-19, isolation precautions including the use of surgical mask, gown, gloves and eye protection were initiated. Nasopharyngeal and oropharyngeal swabs were sent for influenza A/B PCR, a rapid multiplex PCR panel (BioFire FilmArray Upper Respiratory Pathogen Panel, Biomerieux, Salt Lake City, Utah), and a SARS-CoV-2 PCR in a reference laboratory since institutional testing was not yet available. Influenza PCR was negative within hours. The next day, results of the multiplex PCR panel were available in the electronic medical record (EMR). No pathogens were detected, including the common coronaviruses HCoV-229E, HCoV-OC43, HCoV-NL63 and HCoV-HKU1 (Figure 1). The clinical team saw the results of negative coronaviruses and interpreted this as negative testing for COVID-19 and stopped isolation precautions. The next morning, the infection prevention team clarified with the clinical team that SARS-CoV-2 testing was still pending, and isolation precautions were resumed. Subsequently, the SARS-CoV-2 PCR was negative and isolation precautions were definitively discontinued.

Advanced diagnostic testing can be useful, but can also be confusing or harmful without appropriate education or clinical support for interpretation [3]. In this case, results from a rapid respiratory diagnostic panel were interpreted incorrectly, leading to premature discontinuation of isolation precautions, and heightened staff anxiety about possible exposure. This case presents an important learning opportunity for healthcare providers and facilities using similar testing. Interpreting advanced diagnostic tests in the setting of a novel pathogen such as SARS-CoV-2 poses two major challenges. First, clinicians frequently send these tests in patients with suspected COVID-19 to identify alternative pathogens. However, even if another virus is found, the safety of discontinuing isolation precautions remains unclear as co-infections with SARS-CoV-2 and other viruses may occur [4]. Second, clinicians can 


\begin{tabular}{|c|c|}
\hline \multicolumn{2}{|l|}{ Upper Respiratory Pathogen Panel-URPP } \\
\hline Upper Respiratory Pathogen Specimen & *NP \\
\hline Adenovirus-URPP & Not Detected \\
\hline Coronavirus 229E-URPP & Not Detected \\
\hline Coronavirus HKU1-URPP & Not Detected \\
\hline Coronavirus NL63-URPP & Not Detected \\
\hline Coronavirus OC43-URPP & Not Detected \\
\hline Metapneumovirus-URPP & Not Detected \\
\hline Enterovirus/Rhinovirus-URPP & Not Detected \\
\hline Influenza A-URPP & Not Detected \\
\hline Influenza B-URPP & Not Detected \\
\hline Parainfluenza 1-URPP & Not Detected \\
\hline Parainfluenza 2-URPP & Not Detected \\
\hline Parainfluenza 3-URPP & Not Detected \\
\hline Parainfluenza 4-URPP & Not Detected \\
\hline Respiratory Syncytial Virus-URPP & Not Detected \\
\hline Bordetella pertussis-URPP & Not Detected \\
\hline Chlamydophilia pneumoniae-URPP & Not Detected \\
\hline Mycoplasma pneumoniae-URPP & Not Detected \\
\hline
\end{tabular}

Figure 1: Results of the rapid respiratory diagnostic panel. A negative multiplex PCR respiratory panel in the general laboratory section of the electronic medical record. Note that the four common human coronaviruses are listed by name. The interpretation result (not shown) which explains that SARS-CoV-2 is not tested is obtained by clicking on the specimen result and then the comment tab.

easily misinterpret the multiplex PCR results of "coronavirus" for COVID-19, particularly since the SARS-CoV-2 PCR test, at that time, took much longer to result than the multiplex PCR panel.

Modifying how results are presented in the EMR can help with diagnostic stewardship and test interpretation [5]. For rapid molecular diagnostics, institutions often add comments in the EMR to clarify result interpretation or chose to selectively suppress organisms not considered clinically relevant [5]. Weighing the relatively new availability of this multiplex PCR, the ability to quickly change EMR reporting, and laboratory regulatory requirements, our laboratory leadership decided to add a comment in the EMR interpretation section of the multiplex PCR indicating that COVID-19 was not tested. However due to EMR constraints, this comment required two additional clicks to view and was not seen by the clinical team. Subsequently, this case prompted the addition of an EMR log-in pop-up explaining that the multiplex PCR did not include COVID-19.

In this case the premature discontinuation of isolation precautions was multifactorial. While human error was the direct cause, the lack of guidance in interpreting the test contributed to the decision to discontinue isolation. Fortunately, the clinical team's electronic order to discontinue isolation precautions automatically triggered a review by our infection prevention team which revealed the outstanding COVID-19 testing and prompted resumption of precautions. This underscores the need for automated checks in medicine, especially in the time of pandemic, as well as consistent documentation regarding isolation precautions.

Healthcare facilities continue to face challenges with adding testing capacity and results, particularly in a rapidly changing environment such as a pandemic. Clinicians, infection preventionists, information technologists and laboratory departments should work together to ensure clarity in reporting results, especially as testing for SARS-CoV-2 will likely be added to multiplex PCR panels in the future [6]. While this near miss did not cause direct patient harm, it resulted in avoidable staff anxiety, and illustrates both the complexity of medical care delivery and how tests meant to improve diagnostic certainty may have unintended consequences.

Research funding: Jessica Howard-Anderson was supported by an Antibacterial Resistance Leadership Group fellowship [National Institute of Allergy and Infectious Diseases UM1AI104681]. The content is solely the responsibility of the authors and does not necessarily represent the official views of the National Institutes of Health.

Author contributions: All authors have accepted responsibility for the entire content of this manuscript and approved its submission.

Competing interests: Authors state no conflict of interest.

\section{References}

1. Chen Y, Liu Q, Guo D. Emerging coronaviruses: genome structure, replication, and pathogenesis. J Med Virol 2020;92:418-23.

2. CDC COVID-19 Response Team. Severe outcomes among patientswith coronavirus disease 2019 (COVID-19) - United States, February 12-March 16, 2020. MMWR Morb Mortal Wkly Rep 2020; 69:343-6.

3. Morgan DJ, Malani P, Diekema DJ. Diagnostic stewardshipleveraging the laboratory to improve antimicrobial use. J Am Med Assoc 2017;318:607-8.

4. Kim D, Quinn J, Pinsky B, Shah NH, Brown I. Rates of Co-infection between SARS-CoV-2 and other respiratory pathogens. JAMA 2020; 323:2085-6.

5. Messacar K, Parker SK, Todd JK, Dominguez SR. Implementation of rapid molecular infectious disease diagnostics: the role of diagnostic and antimicrobial stewardship. J Clin Microbiol 2017;55: 715-23.

6. Hinton D. FDA letter to Kristen Kanack [internet]. Place unknown: publisher unknown. [updated 2020 May 6; cited 2020 June 23]; 2020. Available from: https://docs.biofiredx.com/wp-content/ uploads/BFR0000-9068-RP2.1-EUA-Authorization-Letter-2.pdf. 